\title{
Herméneutique et joueur modèle : Interpréter le mythe et le fait religieux dans la série Soulsborne de From Software
}

Hermeutic and ideal player: Interpreting myth and religious fact in From

Software's Soulsborne series

\section{Yannick Kernec'h et Clément Personnic}

\section{OpenEdition \\ Journals}

Édition électronique

URL : https://journals.openedition.org/sdj/3273

DOI : $10.4000 /$ sdj.3273

ISSN : 2269-2657

\section{Éditeur}

Laboratoire EXPERICE - Centre de Recherche Interuniversitaire Expérience Ressources Culturelles

Education

\section{Référence électronique}

Yannick Kernec'h et Clément Personnic, « Herméneutique et joueur modèle : Interpréter le mythe et le fait religieux dans la série Soulsborne de From Software ", Sciences du jeu [En ligne], 15 | 2021, mis en ligne le 27 juin 2021, consulté le 26 novembre 2021. URL : http://journals.openedition.org/sdj/3273 ; DOI : https://doi.org/10.4000/sdj.3273

Ce document a été généré automatiquement le 26 novembre 2021.

\section{(c) (1) (9)}

La revue Sciences du jeu est mise à disposition selon les termes de la Licence Creative Commons Attribution - Pas d'Utilisation Commerciale - Pas de Modification 4.0 International. 


\title{
Herméneutique et joueur modèle : Interpréter le mythe et le fait religieux dans la série Soulsborne de From Software
}

\author{
Hermeutic and ideal player: Interpreting myth and religious fact in From \\ Software's Soulsborne series
}

Yannick Kernec'h et Clément Personnic

1 De Demon's Souls (2009), précurseur estimé, à la trilogie Dark Souls (2011-2016) et Bloodborne (2015), l'entreprise japonaise de développement de jeux vidéo From Software a su développer sur une demi-douzaine d'années une véritable série, communément appelée «Soulsborne », ${ }^{1}$ fondée sur des échos ludiques ou mythologiques. La dimension sérielle des Soulsborne pose le problème d'une conception contemporaine de la sérialité - en raison notamment du succès des séries télévisées et de leur caractère ultrafeuilletonnant - qui fait prévaloir la dimension narrative au détriment d'autres axes d'études. C'est par extension l'idée d'une sérialité vidéoludique non narrato-centrée, « qui ne passerait ni par le prisme de la "mise en scène ", ni par celui de la narration " (Saute-Requin, 2019) que nous désirons défendre en qualifiant ainsi le signifiant Soulsborne de "série». À ce titre, nous choisissons d'écarter Sekiro: Shadow Die Twice (From Software, 2019) de cette étude - dernier jeu en date de Hidetaka Miyazaki et créateur de la série Soulsborne. En effet, si l'on retrouve dans ce titre une philosophie semblable de la difficulté ou de l'échec ludique, il n'en demeure pas moins profondément distinct des opus précédents de la série compte tenu de la présence d'une mécanique d'infiltration, d'une progression aérienne, d'un système de développement de personnage amputé des traditionnelles données statistiques, et d'une relative clarté de son récit.

2 Si le succès de la série est aujourd'hui indéniable le lancement a été difficile en raison de la dimension énigmatique du titre, aussi bien du côté de la jouabilité que de son caractère radicalement distinct des productions de son époque ; en témoigne le 
préambule de la critique du remake de Demon's Souls (Bluepoint Games, 2020) sur le site gamekult :

Quand Demon's Souls s'est montré pour la première fois au Tokyo Game Show 2008, on n'imaginait pas découvrir sous nos yeux l'un des jeux les plus importants de l'histoire. Rétrospectivement, la preview de l'époque est à se rouler par terre de honte, allant jusqu'à parler de possible " cadeau empoisonné » pour Sony, qui soit dit en passant ne savait pas trop quoi en faire non plus. Dans la moiteur d'un salon, il fallait faire preuve d'un sixième sens pour imaginer qu'un titre aussi lourd, lent et en marge de la production de l'époque puisse imposer ses propres codes et remettre en avant la notion d'exploration et de persévérance dans le genre de l'actionaventure. $^{2}$

3 Dans un article qualifiant Demon's Souls de jeu le plus important de la décennie, le site d'actualité vidéoludique Polygon rend compte de la singularité du titre par son " opacité », sa "difficulté » et des choix « antithétiques » avec les «conventions de game design" de l'époque. ${ }^{3}$ Ils renvoient notamment dos à dos cette production à l'approche, qualifiée d'» hollywoodienne », d'Uncharted (Naughty Dogs, 2007-2017), Mass Effect (Bioware, 2007-2017) ou encore Assassin's Creed (Ubisoft, 2007-2020). Par le qualificatif « hollywoodien » - qui se rapportent autant à des standards narratifs qu'aux budgets faramineux engagés sur ces productions filmiques - l'auteur oppose ainsi la surdramatisation et l'accessibilité de telles productions à l'exposition minimale ainsi que la narration par l'environnement (Jenkins, 2004) omniprésente dans la série de From Software (Calafat, 2018). Autrement dit, l'histoire de la série Soulsborne est entièrement racontée par le biais de l'exploration des lieux par le personnage.

Durant son activité ludique, le joueur est sans cesse mis en rapport avec des éléments diégétiques se rapportant à des mythes et à la sphère du religieux. Des architectures influencées par des bâtisses réelles comme la cathédrale de Milan ${ }^{4}$ aux interactions avec des personnages non joueurs (PNJ) membres d'organisations cléricales, en passant par la manifestation tangible de miracles dans la réalité diégétique par le biais de sorts magiques, From Software en constitue des composantes essentielles du «lore », c'est-àdire les soubassements de leur univers fictionnel. Pourtant, nous ne semblons jamais vraiment en mesure d'en discerner l'organisation globale.

Comment procéder pour mettre en lumière une telle déficience ? Considérons dans un premier temps la structure psycho-cognitive du joueur, c'est-à-dire le processus par lequel il structure les données diégétiques d'un jeu vidéo en général, puis de la série des Soulsborne en particulier. Dominic Arsenault et Bernard Perron (2009, pp. 109-131) proposent un modèle de compréhension de l'activité ludique centré autour du joueur au moyen de trois spirales - une spirale heuristique de la jouabilité, une spirale heuristique de la narration et une spirale herméneutique - représentant la progression du joueur au fil de sa pratique ludique, qui voit ses compétences, sa compréhension du récit et sa capacité à l'interpréter s'élargir au fil du temps. La difficulté de raccorder ces éléments impliquerait notamment d'attribuer à cette spirale herméneutique un rôle prépondérant dans la reconstitution du tissu diégétique.

6 Si cette architecture psycho-cognitive est universalisable, il est néanmoins nécessaire de postuler un type de joueur dont l'attitude ludique viserait à appréhender la diégèse des Soulsborne et à en interpréter les données fictionnelles. La théorie du " play design » s'avère de fait pertinente afin d'expliciter la méthode par laquelle le joueur devient «qualifié » (Genvo, 2008) pour terminer le jeu et se figurer la mythologie, en ce que « cela permet de décrire la façon dont un système de règles est configuré pour faire 
vivre à un certain “ joueur-modèle " une expérience de jeu singulière " (Genvo, 2008). Nous devons par conséquent dresser un portrait du «joueur modèle» de cette série. Ainsi, à partir des propos du concepteur Hidetaka Miyazaki et de notre propre expérience, nous allons tenter dans un premier temps de déterminer le parcours perceptivo-cognitif qui serait celui du joueur modèle des Soulsborne, préalable nécessaire à un examen en profondeur de la dimension mythique et du religieux de la série.

\section{Vers un joueur modèle des Soulsborne}

7 Le joueur modèle incarne cette idée d'un joueur conçu comme un récepteur idéal des intentions de game design d'une instance créatrice, c'est-à-dire une "pratique de conception » ayant ses « propres principes essentiels, un système d'idées qui définit ce que sont les jeux et comment ils fonctionnent " (Salen et Zimmerman, 2004, p. 6). Un pareil contenu - aussi bien thématique, narratif que diégétique - est livré au joueur par un ensemble de techniques auxquelles celui-ci doit se montrer attentif: narration environnementale prégnante, usage parcimonieux de scènes cinématiques ou autres événements intradiégétiques. Or, la construction du monde - ce qui désigne les moyens par lesquels le lore, notamment, est élaboré - résulte de choix de game design. De fait, le joueur doit adopter une attitude ludique (Genvo, 2008) qui le prédispose à organiser ces éléments.

8 Comme le rappelle Genvo, le concept de « joueur modèle » est directement issu d'une théorisation d'Umberto Eco selon laquelle :

Un texte est considéré par Eco comme un tissu de "non-dits ", qui doivent être actualisés lors de l'actualisation de contenu. Dans cette optique, un texte est émis pour quelqu'un ayant les « compétences » de l'actualiser, même si la compétence du destinataire n'est pas forcément celle de l'émetteur. «Donc prévoir son Lecteur Modèle ne signifie pas uniquement "espérer" qu'il existe, cela signifie aussi agir sur le texte de façon à le construire. Un texte repose sur une compétence, mais, de plus, il contribue à la produire ». (Genvo, 2008).

9 Le joueur modèle, si tant est qu'il existe, n'est donc pas donné mais "espéré ». Il suppose une "coopération » entre un design de jeu conçu pour délivrer une certaine expérience et un joueur réceptif à la proposition qui jouera en conséquence. Cependant, en pratique, comment étudier un tel joueur paradigmatique si celui-ci n'est pas énoncé clairement?

10 Une telle question revient fondamentalement à interroger la distinction entre joueur et chercheur. En effet, afin d'amasser des connaissances ludiques, il appartient au chercheur de jouer aux jeux; autrement dit, il faut conférer à l'acte de jouer une dimension méthodologique en vue de «se construire une base solide de connaissances sur son objet d'étude » et de les «traduire en connaissance scientifique » (Di Filippo, 2012, p. 179). Plus particulièrement, en ce qui concerne notre propre recherche, il est nécessaire qu'un joueur modèle existe, et que nous le déterminions dans un premier temps, pour rendre compte de l'organisation fictionnelle des mondes des Soulsborne afin que le chercheur puisse étudier ces données ainsi rassemblées - ce qui nous permettra, dans un deuxième temps, d'évaluer les fonctions et les puissances du religieux et du mythe au sein du tissu diégétique synthétisé par le joueur. 
11 Le créateur de la série, Hidetaka Miyazaki, n'est pas avare en commentaires sur sa vision du jeu et sur ses influences; ce sont autant de précieux indices nous permettant de dresser le profil du joueur modèle espéré :

Mais le concept principal derrière le système de mort est l'essai et l'erreur. La difficulté est élevée, mais toujours surmontable. Tout le monde peut y arriver sans trop de technique - tout ce dont vous avez besoin est d'apprendre de vos morts, comment surmonter les difficultés. Surmonter les défis en apprenant quelque chose dans un jeu est un sentiment très gratifiant, et c'est ce que j'ai voulu prioriser dans Dark Souls et Demon's Souls. Et grâce au jeu en ligne, vous pouvez même apprendre quelque chose de la mort de quelqu'un d'autre. Je dirais que c'est le concept principal derrière le jeu en ligne également. ${ }^{5}$

D'un point de vue strictement ludologique, Miyazaki désire créer un jeu opposant de la résistance au joueur, qui ne donne pas de sentiment d'injustice, mais qui au contraire inviterait à la progression au travers d'un apprentissage par la répétition (clément, 2013, pp. 49-63), mais aussi de l'entraide (grâce au mode multijoueur notamment). Par conséquent, le joueur modèle des Soulsborne serait avant tout un joueur qui embrasse une certaine philosophie de la difficulté, qui monte en compétences et en connaissances.

Outre la jouabilité et les questions liées à la difficulté, Miyazaki est aussi un auteur qui réinjecte une expérience passée particulière. Comme le rapporte The Guardian, il tente de recréer les sensations de lecture de son enfance :

Quand Hidetaka Miyazaki était enfant, il était un fervent lecteur, bien que peu talentueux. Souvent, il arrivait à des passages de textes qu'il ne pouvait comprendre, alors il permettait à son imagination de combler les blancs à l'aide des illustrations qui les accompagnaient. De cette façon, il avait la sensation de coécrire la fiction au côté de son auteur original. Le frisson de ce processus ne l'a jamais quitté - et est très présent dans ses jeux vidéo fascinants et ésotériques. ${ }^{6}$

Hidetaka Miyazaki souhaite ainsi rendre son univers cryptique et quasi insaisissable pour le joueur. On le remarque notamment avec ce choix constant d'une narration indirecte, par l'environnement, des descriptions d'inventaire floues et une utilisation très restreinte des cinématiques. La difficulté de synthétiser cette diégèse serait ainsi issue, en terme créatif, d'une volonté manifeste de brouiller les pistes et de faire de l'utilisateur, l'acteur d'une telle reconstitution. Miyazaki conçoit ses jeux de manière à anticiper un joueur modèle qui serait non seulement capable de surmonter la difficulté du titre, mais aussi de synthétiser des éléments fictionnels abondants et épars. Cependant, cette double anticipation du joueur modèle pose un problème de taille: celui de la possibilité d'une conciliation entre ces deux attitudes ludiques distinctes. Peuvent-elles seulement fonctionner ensemble ?

Notre propre expérience de joueur s'est notamment heurtée un temps à cette difficulté, la prise en main et la compréhension des mécaniques faisant office de première nécessité. En effet, tout dispositif vidéoludique place d'emblée le joueur dans une posture de réaction face à ce qui lui est exposé : «[...] nous argumenterions plutôt qu'un jeu vidéo est une chaîne de réactions. Le joueur n'agit pas autant qu'il réagit à ce que le jeu lui présente et de façon similaire, le jeu réagit à ses commandes » (Arsenault et Perron, 2009, p 119-120). La découverte de ces jeux implique en premier lieu de réagir à ce que le jeu nous soumet, de se confronter à la maîtrise des mécaniques, la compréhension des objectifs et au développement d'un niveau de compétence suffisant - ce que Sébastien Genvo (2008) décrit comme les quatre «modalités du faire » dans 
l'application qu'il propose du schéma narratif canonique de Greimas au jeu : le devoirfaire, le vouloir-faire, le pouvoir-faire et le savoir-faire.

De fait, nous améliorons nos compétences en comprenant au fil du jeu ce que nous devons faire et comment y parvenir (améliorer notre personnage, combattre efficacement, trouver des équipements qui faciliterait notre progression, etc). Cependant, au terme de l'aventure, il subsiste une interrogation de taille : qu'est-ce qu'un jeu Soulsborne nous a raconté ? Que pouvons-nous dire à propos de l'univers fictionnel ? En pratique, certains éléments n'ont pas été compris et il a fallu différents moyens (rejouabilité, recherches Internet, etc.) pour faire face à la difficulté et appréhender nombre d'éléments diégétiques. Du point de vue du chercheur, ce constat est similaire à celui établi par Laurent Di Fillippo lorsqu'il appose un regard rétrospectif sur son expérience de joueur et la nécessité de sa liaison avec son activité de chercheur :

Un autre point important, dont je me suis rendu compte par la pratique du jeu, est que je ne remarquais certains éléments pertinents pour mes recherches qu'après avoir reproduit plusieurs fois la même opération. Le jeu demandant de la concentration et de la maîtrise pour effectuer certaines tâches, il est souvent impossible de voir tous les éléments d'une scène. Un combat, par exemple, demande de réagir très vite dans l'action. C'est pourquoi il est nécessaire au chercheur de faire et de refaire le jeu plusieurs fois s'il veut atteindre la saturation de manière qualitative (Di Fillippo, 2012, p. 182).

Néanmoins, lorsque nous considérons notre propre expérience de joueur, certaines interprétations que nous avons formulées n'ont pas émergé dans le cadre de la présente recherche, mais lui ont bel et bien préexisté. Lors de nos premières sessions, ce n'est pas tant que nous avons manqué des éléments dans le but de rendre compte scientifiquement de ces procédés interprétatifs, car nous ignorions que ces matériaux pussent être remobilisés ultérieurement dans un cadre scientifique; ce sont plutôt les éléments diégétiques sur lesquels nous avions fait l'impasse lors de premières parties qui, par l'ostensible vide diégétique que leur absence entraînait, nous exhortait à les récolter, loin de toute démarche de recherche ; rien de moins, finalement, qu'un appel à la complétude fictionnelle, ou encore à la reconstitution de la diégèse. En cela, on peut affirmer que la conception de jeu pousse son joueur à rejouer pour comprendre et interpréter.

Afin de comprendre cet apparent paradoxe issu de la montée en compétence ludique au détriment de notre connaissance de la diégèse, revenons plus précisément sur le modèle des spirales de jouabilité proposé par Dominic Arsenault et Bernard Perron. Ceux-ci proposent en effet d'envisager l'acte ludique non plus seulement comme un cercle, à l'instar de Johan Huizinga et sa notion de « cercle magique » (Huizinga, 1988 [1938]), mais à la manière d'une activité cyclique représentée sous la forme d'une combinaison de trois spirales. Une première, qualifiée de spirale heuristique de la jouabilité, concerne les compétences et les savoirs du joueur sur le jeu, dans son caractère le plus ludique, détaché des éléments narratifs et diégétiques. La spirale heuristique de la narration, quant à elle, est "contenue au sein de la jouabilité » et "marque les événements narratifs qui se déroulent au travers du jeu» (Arsenault et Perron, 2009, p.116). Enfin, la spirale herméneutique représente la dimension interprétative de l'effort ludique: "la circularité entre le tout et les parties » qui «mène à la question de l'interprétation et des significations» (p.117). La représentation de ce modèle a pour avantage de prendre en compte la temporalité du 
joueur et, ainsi, d'appuyer l'idée que les compétences, connaissances et interprétations s'acquièrent sur une certaine durée.

Si l'on applique ce modèle à nos premières expériences de ces jeux, on peut dire que notre spirale heuristique de la jouabilité s'est agrandie de façon conséquente, tandis que notre spirale heuristique de la narration est restée minimale : la progression vers la fin des jeux apporte davantage de questions que de réponses. Au bout de notre première aventure, notre spirale herméneutique, quoique plus large que celle de la narration, n'a pas pris beaucoup d'ampleur. Les éléments diégétiques sont en général intégrés de façon peu explicite et toujours parcellaire. Bloodborne, notamment, dissémine un certain nombre de messages ayant pour vocation de livrer des indices au joueur sur les phénomènes se déroulant autour de lui. «Regardez ! Un ciel PâleSang »: ce message que l'on peut lire au sol dans l'un des environnements du jeu est assez remarquable ; sans la définition du terme "PâleSang", le joueur est simplement informé d'une particularité qui toucherait le ciel, sans pour autant en connaître la signification.

Durant la partie, nous amassons de fait quantité d'informations, tout en espérant recueillir plus tard des éléments permettant d'éclaircir ce genre d'incompréhension. Toutefois, le rythme du jeu et l'action rendent difficile la mémorisation des matériaux fictionnels afin d'en tirer une interprétation satisfaisante. Jacques Henriot soulevait déjà cette difficulté à penser et jouer le jeu ; Bernard Perron l'appuie :

Il reste conscient de la position qui l'amène à jouer moins pour en parler mieux, et prend même "quelque plaisir à insister sur ce que l'obligation a de paradoxal » (Henriot, 1989a, p. 11). Dans la mesure où il se pose lui-même en joueur pour écrire, Henriot ne dit pas qu'il ne faut pas en soi jouer, ni qu'il ne faut pas connaitre le plus possible le jeu (qui n'est pas un jeu ou un type de jeux en particulier). Il n'y a de toute façon «Pas de jeu sans jouer ; pas de jouer sans joueur » (Henriot, 1989a, p. 108). On ne passe pas 25 ans à lire, réfléchir, considérer et interroger ce qu'est jouer, donc "agir pour réfléchir sur l'action» (afin de reprendre l'expression critique de Duflo, 1997, p. 32), si l'on ne connait pas bien ce dont on parle. On ne peut tout simplement pas faire les deux en même temps (Perron, 2013, § 8).

Pour étudier le jeu, il faudrait poser la manette. On pourrait faire un constat similaire pour le joueur interprète. Le jeu est exigeant et met régulièrement l'utilisateur dans des situations périlleuses à même d'entraîner un game over, ce qui place l'enjeu de la survie au premier plan et l'opération d'interprétation - c'est-à-dire le processus herméneutique désigné par Arsenault et Perron - au second. Il y a ici un parallèle possible entre la posture du chercheur et celle d'un joueur cherchant à percer les mystères de la narration des titres de From Software. Les combats de boss illustrent cette difficile conciliation où, à l'image de ce que disait Henriot, la « réflexion paralyse l'action » et « l'action tue la réflexion ».

Prenons à titre d'exemple la confrontation avec le chef (boss) Astrée la sixième sainte dans Demon's Souls, qui consiste à pénétrer dans un sanctuaire et à abattre un démon d'apparence humaine. Le combat débute par la traversée d'un brouillard séparant l'avatar de la zone du chef - ce qui, dans la série Soulsborne, constitue en soi une ritualisation interactive des conflits majeurs. ${ }^{7}$ Une séquence cinématique se lance alors, dévoilant partiellement Astrée et son statut de boss qui est signifié au joueur par l'apparition d'une barre de vie en bas de l'écran. Alors que celle-ci demeure immobile, un second ennemi, Garl Vinland, fait obstruction. Une fois celui-ci défait, la barre de santé d'Astrée est toujours apparente : l'affrontement n'est donc pas achevé. Le joueur 
en vient ainsi à s'approcher du chef qui, bien loin d'adopter une posture agressive augurant une joute à venir, met fin par lui-même à sa barre de vie et disparaît. Un tel comportement correspondrait ludiquement à un abandon et, d'un point de vue fictionnel, à un suicide ; d'une certaine manière, le combat annoncé n'a pas lieu; ou plutôt, il n'aura pas lieu à la première tentative, car les étapes menant à cette résolution du combat sont celles que le joueur inexpérimenté peut suivre lors de la première rencontre. Précisons que si le joueur en vient à éviter le garde du corps et attaque d'emblée Astrée en début de combat se défend contre notre agression. Une telle séquence de jeu nous révèle que les chefs ont eux aussi des motivations qui ne sont pas dévoilées explicitement, mais sous-entendues par le texte.

Lorsque Sainte Astrée est présentée, ainsi exposée à la vue de ceux qui l'idolâtrent, l'ordre qu'elle donne à son champion n'aide en rien à appréhender les tenants et les aboutissants de la manifestation d'ordre liturgique en cours. De plus, la cinématique, inusuelle au sein du titre, ne contextualise pas les corps des chevaliers missionnés visibles dans ce lieu sacré. L'immédiateté de l'action ne la rendant pas vraiment possible, la réflexion sur cette séquence de jeu prend place a posteriori. De fait, ce combat de boss organise des éléments diégétiques que l'impératif d'action (Genvo, 2011, p. 98) vient intriquer. En conséquence, notre spirale heuristique de la jouabilité, pour reprendre le modèle établit par Arsenault et Perron, progresse et s'élargit beaucoup plus rapidement que la spirale heuristique de la narration ou que la spirale herméneutique.

En continuant de monter en compétence par la répétition et la rejouabilité, cette opération cognitive de reconstitution du tissu diégétique s'est simplifiée, puisque nous avons intégré des "séries de schémas procéduraux" et acquis les "capacité[s] nécessaire[s] pour un contrôle volontaire sans effort » (Grodal, 2000, p. 204). Ces acquis ont permis de mieux réfléchir en jeu et en dehors du jeu, afin d'arriver à une compréhension personnelle, mais basée sur les informations internes du jeu. Notre expérience ludique nous conduit ainsi à penser que notre profil de joueur a rencontré celui souhaité par le concepteur. En effet, la montée en compétence nous a progressivement permis d'établir des interprétations de la diégèse plus qualitatives en raison d'une pression sur notre activité de jeu de moins en moins importante. Notons que, nos interprétations ont par ailleurs également été nourries des réflexions d'autres utilisateurs qui ont partagé leurs propres théories sur Internet. La communauté des joueurs des Soulsborne est très active dans la production d'explications concernant la diégèse du jeu ; ce que des joueurs ne remarquent pas est bien souvent relevé par d'autres.

La constitution d'un tel joueur modèle pour la série Soulsborne nous permet de considérer la dimension herméneutique comme primordiale, tant d'un point créatif visé par le concepteur - que ludique - comme moteur dans l'activité du joueur. Ce caractère interprétatif n'est pas incompatible avec la difficulté de l'œuvre, cependant elle en ralentit la compréhension du lore. L'information, celle-là même qui oriente l'interprétation, devient en soi une récompense (Labrude, 2018). En cela, l'herméneutique, bien qu'optionnelle dans le modèle de Arsenault et Perron - dans le sens où l'on peut toujours jouer sans interpréter - semble faire partie intégrante de la particularité des Soulsborne. La narration renforce par ailleurs ce sentiment en ce que les différents partis pris semblent amener le joueur à des questionnements multiples et perpétuels sur le monde; et la matière privilégiée pour interpréter ces univers sériels 
se rapporte, selon nous, aux dimensions du mythe - en première instance - et du religieux.

\section{Le joueur modèle face au fait religieux}

Tout au long de son parcours, le joueur est amené à interagir avec une multitude d'objets, de lieux et de boss. L'ensemble de leur désignation se caractérise par un enchevêtrement assez hétéroclite de signifiants renvoyant très fréquemment à la sphère du religieux. Nous avons ici tenté de les regrouper :

- Divinités (telles que Gwen, dieu de Lumière Solaire ou Velka, déesse du péché pour Dark Souls) ou êtres quasi transcendants (comme l'Ancien dans Demon's Souls ou les Grands de Bloodborne).

- Organisations ecclésiastiques (telles que l'Église du Remède et les Pèlerins des Ténèbres).

- Fonctions sacerdotales (Pontife, Vicaire, Diacres, Archidiacres, Sœur, Père, Prêtres, Oracle, Rédempteur et Paladin).

- Qualificatifs sacralisateurs (Martyr, Saint(e), Vierge ${ }^{8}$, Fidèles, Pèlerins, profanée, Damnés, miracle et divin[e]).

- Lieux/objets/espaces de culte (Paroisse, Cathédrale, Chapelle, Cimetière, Tombes, Catacombes et Autel).

- Renvois à des rituels ou procédés liturgiques (processions religieuses, actes de prière, Sacrifices et Crucifixion).

Cette classification met en valeur un champ lexical particulièrement vaste renvoyant aux soubassements cultu(r)els d'une religion au sens durkheimien; en d'autres termes " un système de croyances et de pratiques relatives à des choses sacrées, c'est-à-dire séparées, interdites, croyances et pratiques qui unissent en une même communauté morale, appelée Église, tous ceux qui y adhèrent » (Durkheim, 1991, p. 109) et qui paraît déterminer en grande partie l'ensemble des univers fictionnels des Soulsborne.

Ainsi, à l'orée de sa première partie, le joueur s'avère doublement profane :

- Symboliquement, il l'est au niveau fictionnel, par sa relative ignorance, ou méconnaissance du lore des jeux et, à l'échelle de la série, de sa mythologie, conçue ici dans son rapport narratologique à la sérialité comme "rapport causal, chronologique » entre les épisodes « constitu[ant] les séquences d'un ensemble plus vaste, une structure narrative seconde » (Favard, 2015, p. 142) ; corrélativement, au niveau ludique, par sa méconnaissance des mécaniques de jeu réitérées - ou parentes - d'un opus à l'autre.

- Littéralement, il l'est également au sens où son avatar arpente des territoires marqués par une intense religiosité pour laquelle il n'a pas suivi d'initiation préalable. D'un point de vue plus phénoménologique, cela correspondrait, pour le joueur, à une expérience areligieuse d'un monde fictionnel privé du sens que lui confèrerait le sacré, « réalité absolue [...] qui transcende ce monde-ci, mais qui s'y manifeste et, de ce fait, le sanctifie et le rend réel » (Eliade, 1965, p. 171).

Par ailleurs, on constate que la sémantique déployée afin de caractériser les cultes fictionnels renvoie largement aux religions abrahamiques monothéistes ; Saint Urbain, PNJ de Demon's Souls, entend par exemple la voix de Dieu s'adresser à lui. De manière plus manifeste, le dialogue avec la vendeuse crasseuse, marchande du monde cinq de Demon's Souls (le "Val Fangeux») contient une référence intertextuelle explicite au récit biblique en comparant Astrée avec la Vierge Marie: «Tous les hommes la 
vénèrent comme si elle était la Vierge Marie. Les mêmes hommes qui m'arracheraient mes propres enfants ». Toutefois, ce sont des polythéismes qui ont cours tout au long de la série - dans Demon's Souls (l'Ancien, le Dieu Dragon), dans Dark Souls (et la présence d'un véritable panthéon: Gwen, Gwenevere, Fina, Gwendolin Le Soleil Noir...) et dans Bloodborne (les Grands : Oedon, Amygdala ou encore Kos).

\section{Mythologie et travail herméneutique}

À ce tissu religieux fictionnel - conçu dès lors comme une véritable collectivité morale déterminée par la pratique de rituels et l'adhérence à des croyances, autrement dit une Église (Durkheim, 1991) - se superposent dans les Soulsborne un ensemble de mythes,

récits fondateurs ou exemplaires qui sont préservés à travers les générations, qui se démarquent par leur élaboration poétique ou par leur mise en scène rituelle, qui sont composés de situations, êtres, événements en dehors des règles réelles et de l'expérience quotidienne de la société en question (Leavitt, 2005, § 1 ).

31 Les mondes fictionnels possèdent ainsi leurs héros et autres demi-dieux, au travers notamment des quatre Chevaliers de Gwen dans Dark Souls, de Ludwig dans Bloodborne ou des rois comme Vendrick (Dark Souls 2) ou Allant (Demon's Souls) ; leurs exploits et épopées passés nous sont restitués par les informations fictionnelles éparpillées au sein des différents niveaux du jeu et participent à la création de tels mythes. Ceux-ci sont notamment véhiculés par la description des objets et magies dans l'inventaire de notre personnage, ou encore par divers dialogues avec les PNJ locaux.

Considérons par exemple l'anneau de l'aîné solaire que le joueur collecte dans le niveau de jeu nommé Anor Londo - domaine où résidait les dieux dans Dark Souls et Dark Souls 3 - et qui correspond en jeu à une augmentation du pouvoir des " miracles ", autrement dit un type de magie fondé sur la caractéristique «foi » de notre personnage, manière de constater l'interpénétration entre les mécaniques de jeu et la religiosité de la dimension fictionnelle. Sa description précise que

le premier né du seigneur Gwen hérita de la puissance solaire et dota ses chevaliers de cet anneau capable de renforcer l'effet des miracles. Le premier né de Gwen était un Dieu de la Guerre, mais son inconscience entraîna la perte des Archives et sa propre déchéance. Aujourd'hui, on ${ }^{9}$ a oublié jusqu'à son nom.

Juxtaposé à d'autres évoquant une trahison lors de la guerre opposant les dieux aux Dragons, ce récit parcellaire aura tôt fait de permettre au joueur-interprète d'établir une corrélation entre ce fils déchu et la destruction systématique des Autels Solaires le représentant, ainsi qu'à l'effacement de son nom du panthéon divin. Et le Fils de ne plus apparaître aux côtés du Père (voir Figure 1). 
Figure 1 : Statue manquante de l'Aîné Solaire aux côtés de Gwen et de Gwenevere

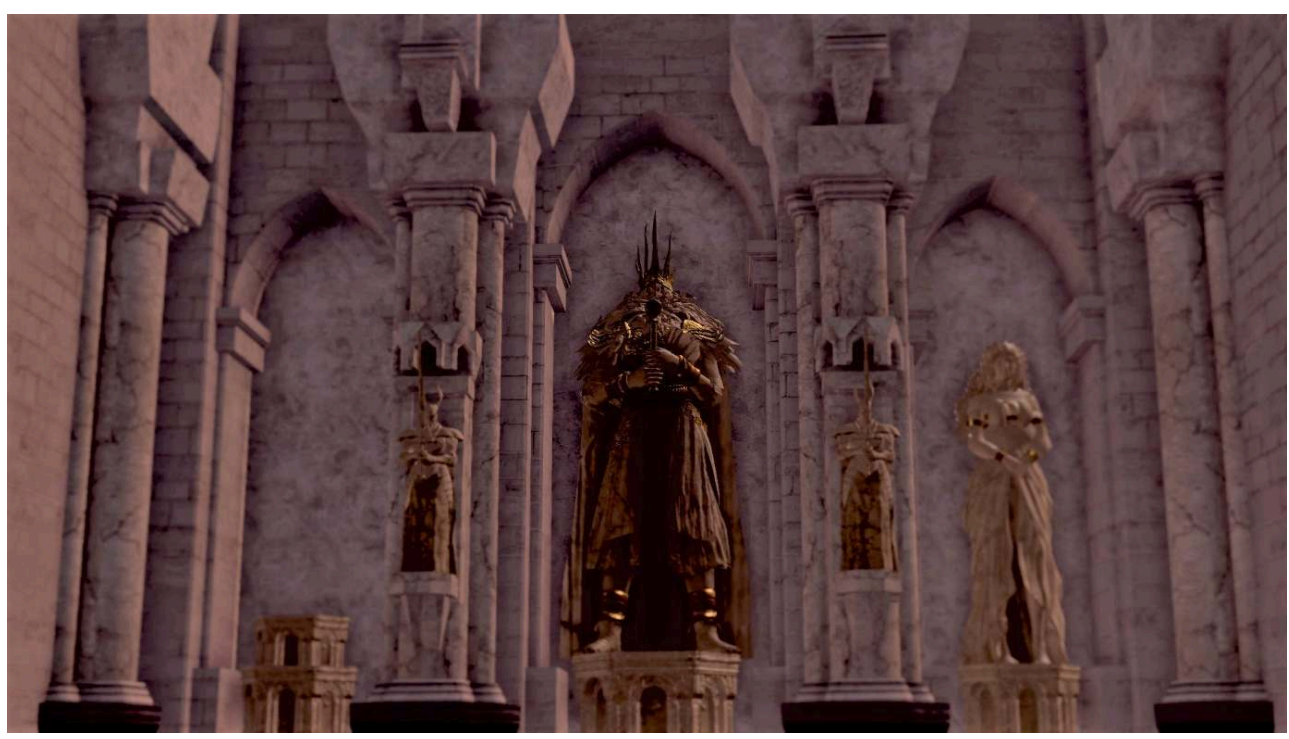

La narration environnementale participe également à l'identification de cette figure divine ayant chu. Dans Dark Souls 3, le niveau optionnel nommé Pic du Dragon Ancien, lieu du culte de la "Voie du Dragon » - correspondant à une religion intradiégétique consacrée au culte des Dragons Anciens et qui se traduit d'un point de vue ludique comme l'appartenance à une faction dans le cadre de l'expérience multijoueurs en ligne - paraît bel et bien accueillir le déshérité et le vénérer. Le joueur peut en effet remarquer, çà et là, des emblèmes liés à ses mythes ainsi qu'une réminiscence de l'autel solaire. Avec ces évocations et l'exposition de statues qui renvoient assez directement à la posture solennelle de Gwen, cet environnement concrétise progressivement la présence d'un être, absent jusqu'à son irruption au premier plan à l'occasion d'un combat de chef. L'ennemi, que le joueur va devoir combattre en deux temps, est armé de la lance d'arrêt de Tueur de Dragons - identifiable sur les autels solaires - et utilise le miracle «lance de foudre "; il y est successivement désigné en sa qualité de Roi des tempêtes durant la première phase de l'affrontement, alors qu'il chevauche un dragon, puis de Roi Sans Nom lors de la seconde phase. Les récompenses à l'issue de l'affrontement - des ensembles d'équipement dont les descriptions appuient sa ressemblance avec Gwen, le premier seigneur - fournissent davantage d'éléments pour permettre au joueur d'interpréter ce chef comme étant le dieu déchu de la légende.

L'ensemble de la série se révèle ainsi exceptionnellement mythogénique, en tant que création continuée de mythes. Le joueur-interprète joue et amoncelle une connaissance brute des mythes, attitude de type mythographique de recensement des mots (descriptions d'objets, dialogues de PNJs...) et des images (symboles, statues, rencontrent directes avec les entités, événements intra cinématiques plus rarement, etc) du texte. Il devient dans une certaine mesure un opérateur mythologique qui actualiserait un monde pour servir sa propre autonarration ; comme le rappelle MarieLaure Ryan :

[...] une représentation proposée comme un récit n'est pas nécessaire pour déclencher le processus cognitif que constitue la narration: nous pouvons former des scripts narratifs dans nos esprits comme une réponse à la vie, ce qui n'est définitivement pas une représentation (bien sûr, nous les expérimentons au travers de processus cognitifs qui produisent des images mentales) (Ryan, 2004, p. 9). 
36 À cet égard, la narration environnementale apparaît ici comme l'outil fondamental de l'herméneute vidéoludique qui, par cette accumulation, prépare le socle de ses futures mises en récit mythologique. Ce constat rend d'autant plus pertinente l'idée de spirale herméneutique comme modèle de représentation de ce processus cognitif - le motif spiralé impliquant en effet un élargissement constant dans le temps. La mythogénie des Soulsborne comporte de fait une dimension programmatique : il s'agirait de « remodeler la fiction» (Labrude, 2018), c'est-à-dire de reconstruire une mythologie comprise comme « système de mythes ou une doctrine sur eux » (Leavitt, 2005).

37 Par ailleurs, on constate que nombre de ces mythes ainsi ordonnancés puisent leur matière dans diverses mythologies païennes - au sens des religions polythéistes de l'Antiquité - qu'elles soient grecques, égyptiennes ou encore mexicaines (Labrude, 2018) ; en termes linguistiques, on dira que les œuvres de From Software contiennent un riche hypertexte ${ }^{10}$ mythique, étant entendu que selon Gérard Genette, «hypertextualité et logique mythique se confondraient et ce n'est plus l'hypertextualité qui permettrait de comprendre la logique mythique, mais le mythe qui viendrait rendre compte de l'hypertextualité » (Rialland, 2005). S'il n'est pas question de mettre en relief l'hypertexte intégral des Soulsborne, nous avons voulu en identifier ici quelques-uns en les mettant en relation avec leur hypotexte d'origine, dans le but de mettre en lumière la dimension herméneutique du travail cognitif du joueur.

38 Le Monde peint d'Ariamis constitue un niveau caché de Dark Souls accessible via une interaction entre l'avatar et la surface d'une peinture sacrée exposée au sein d'un lieu de culte imposant dont la fonction première semble justement de contenir ce tableau. Aux confins de ce tableau-Monde, le joueur fait la rencontre de Priscilla la Métissée, entité que le joueur peut choisir de combattre. Notons que le caractère hypertextuel de cette rencontre n'est concevable par le joueur que s'il a auparavant effectué le travail mythographique - de reconstitution des données diégétiques. Priscilla la Métissée, qui porte sa nature hybride jusque dans son nom, est le fruit de la rencontre entre un dragon blanc et une divinité supposée dont l'origine reste mystérieuse. Cet être réside dans ce qui correspondrait à une prison sous bonne garde - en témoignent les nombreux ennemis, nommés "gardiens de la peinture ", à occire devant le tableau - et l'on peut supposer que c'est son existence même qui est source de discorde. L'analogie avec le mythe grec du Minotaure est manifeste, même s'il existe dans le jeu des représentations plus proche du Minotaure du point de vue strictement esthétique, notamment celle du Démon Taureau. En effet, le Minotaure, monstruosité mi-homme mi-taureau issu de l'union entre Pasiphaé, épouse du roi Minos, et un taureau blanc envoyé par Poséidon, fut enfermé dans le labyrinthe de Dédale afin que nul ne puisse lever le voile sur son existence.

39 La démarche interprétative fictionnelle peut également être poursuivie afin de lever le voile sur l'identité des géniteurs de Priscilla. Le seul dragon blanc connu de la diégèse est Seath l'écorché, un des seigneurs d'Anor Londo ; et il est probable, pour le joueurmythologue, qu'il s'agisse du père. L'identité de la mère est plus obscure. Pour Damien Mecheri et Sylvain Romieu (2017, p. 157), les nombreux objets présents dans le monde d'Ariamis en rapport avec Velka, déesse du péché, laissent entendre une connexion forte avec Priscilla. Une autre interprétation, sans doute plus cohérente que les deux auteurs ne la conçoivent, consiste à voir en Gwenevere la figure maternelle. Cependant et contrairement à ce qu'ils affirment, l'indice selon lequel la comédienne de doublage 
est identique pour les deux personnages ne constitue pas l'unique élément probant. En effet, la narration environnementale semble aussi conforter cette hypothèse. La composition de la zone dans laquelle est accrochée la peinture est assez révélatrice : le tableau est placé face à la statue de la déesse de la fertilité (ce qui porterait à croire que nous nous situons dans l'église de la déesse). En outre, cette dernière deviendra longtemps après - selon la description de l'objet « bénédiction divine » de Dark Souls 3 la femme du Roi Oceiros ; un être fasciné par les dragons au point d'adopter les caractéristiques physiques de Seath, le dragon blanc sans écailles.

Les mythes Abrahamiques enrichissent également l'hypertexte des Soulsborne. La référence au péché originel et à la corruption de l'homme par nahash, le serpent de la Genèse, est patente dans Dark Souls. L'exploration des Ruines de la Nouvelle Londo zone submergée par une action divine pour repousser la menace des Abysses, événement mythique pouvant à son tour renvoyer à celui du Déluge vétérotestamentaire - permet par exemple de s'entretenir avec un Serpent Primordial nommé Kaathe. Ses révélations concernant la portée morale de l'action de l'avatar sur le monde fictionnel peuvent (re)configurer le sens des agissements du joueur et influencer son choix ultime. Une fois le chef (boss) final annihilé, le joueur peut choisir ou non de raviver la Flamme, symbole diégétique lié à la prolongation d'un Âge sur le déclin. Laisser la flamme mourir constituerait dès lors une sorte de voie du Péché, qui renverrait chez Saint Paul à "l'effort pour l'homme de se sauver sans Dieu " (Malet, 1962, p. 207). Son discours s'oppose frontalement à celui de l'autre Serpent, Frampt, que l'on rencontre plus aisément au cours du parcours ludique et qui souhaite voir le joueur accomplir la volonté des dieux - représentation de la voie de la Grâce. Preuve, s'il en est, que le récit n'est pas entièrement absent des Soulsborne ; nous sommes ici informés de la présence d'un conflit ancestral et d'une possible manipulation d'un des partis, ce qui accroît quelque peu, de manière figurée, la spirale heuristique de la narration. Cependant, rien ne précise ce que le joueur doit faire de cette information ni laquelle de ces deux entités ment à cet instant précis : ceci est affaire d'herméneutique.

41 Ces multiples références aux religions bibliques nous exhortent à penser l'articulation, dans la série, entre ce qui relève de la mythologie diégétique, et ce qui a trait à la religiosité de ces univers fictionnels. Si, comme nous l'avancions précédemment, ces deux dimensions semblent inonder l'espace diégétique - à plus forte raison pour le mythologique - leur coexistence demeure paradoxale. En effet, l'Église liée, comme on l'a vu avec Durkheim, à un terreau social vivant, est ici l'expression d'un monde immobile, en état de décrépitude. C'est particulièrement le cas dans la trilogie Dark Souls, le dernier opus nous faisant même entrevoir, dans les ultimes moments du contenu additionnel au jeu original intitulé The Ringed City (From Software, 2017), un monde mort dont il ne subsiste que de stériles étendues de poussière.

A l'inverse, la mythologie est pugnace, vivante, voire incarnée telle qu'en témoigne la participation active des anciens héros, dieux ou même des Grands, et leurs interactions avec l'avatar. Celui-ci est d'ailleurs amené à être mythifié de manière rétroactive à l'occasion des voyages dans le temps mis en jeu dans Dark Souls et son contenu additionnel intitule Artorias of the Abyss (From Software, 2011) ou encore celui de Bloodborne: The Old Hunters (From Software, 2015); en témoigne la description de l'âme du chevalier Artorias, qui met en relief une mythologie évolutive, fonction des actions du joueur, ce « héros inconnu» : 
Chevalier du seigneur Gwen qui a été consumé par les Abysses. La légende décrivant Artorias repoussant les Abysses ne disait pas tout. Il aurait été vaincu et un héros inconnu, véritable vainqueur des Abysses, aurait protégé son honneur.

Une brève comparaison du chapitre I de la Genèse avec les cinématiques d'ordre cosmogonique de Demon's Souls et du premier volet de la trilogie Dark Souls peut nous permettre d'éclaircir ce paradoxe juxtaposant mythe vivant et religion cadavérique. Elles sont, en première lecture, une sorte de Genèse en cinématique qui n'est pas tant, en apparence, la mise en place d'une histoire que celle d'un Monde et de sa cosmogonie. Le lien intertextuel avec la Genèse 1, soit la subdivision de la Création en jours, est à ce titre particulièrement manifeste dans Demon's Souls : » Au premier jour, L'homme reçut une âme et la clairvoyance. Au deuxième jour, La Terre fut victime d'un poison irrémédiable. Un démon mangeur d'âme. "

Le récit mythologique de Dark Souls prend quant à lui racine dans un passé immémorial au sein duquel le Monde a opéré une transition entre un état intemporel, sans divinité au sens que cette série confère au statut divin, sur lequel nous reviendrons - et des cycles, comme le dévoile la voix off narrant l'origine du monde dans la cinématique d'ouverture de Dark Souls:

L'âge des anciens... à cette époque... Le monde n'était encore qu'une ébauche noyée dans le brouillard. Une terre rocheuse grisâtre d'arbres titanesques et Dragons immortels. Vint alors l'avènement du Feu, et avec le feu naquit la diversité. La chaleur et le froid, la vie et la mort, et bien entendu... la Lumière et les Ténèbres. C'est alors qu'ils sortirent des Ténèbres. Et découvrirent dans la Flamme, les âmes des Seigneurs. Nito, le premier d'entre les morts. La sorcière d'Izalith et ses filles du Chaos. Gwen, le Seigneur Solaire, accompagné de ses fidèles chevaliers, et cet être de petite, des plus discrets, qu'on oublie si souvent. Pourvus de la Force des Seigneurs, ils défièrent alors les Dragons. Les éclairs de Gwen fracassèrent leurs écailles de pierre. Les sorcières déchaînèrent contre eux des tempêtes de flammes. Tandis que Nito semait dans leurs rangs les graines de la maladie et de la mort. En trahissant les siens, Seath l'écorché scella le destin funeste des Dragons. Ainsi débuta l'âge du Feu. Mais bientôt l'âge du Feu mourra et laissera place aux Ténèbres. Aujourd'hui la lueur de ses braises ne suffit plus à éclairer la nuit éternelle dans laquelle le monde est plongé. Et des porteurs de la Marque sombre maudite ont fait leur apparition parmi les vivants.

Comme Marc de Launay l'interprète à propos de la Genèse 1, en prenant appui sur la terminologie de Cassirer, ces deux récits cosmogoniques de la série Soulsborne font état de la création de l'homme et semblent coïncider au « passage d'une forme symbolique "mythe " à une autre forme, "religion" " (de Launay, 2010, p. 465), notamment " car dès l'apparition de l'homme, le temps cesse d'être chronologie indifférente, celle des cycles cosmogoniques, pour devenir durée appréciée, histoire» (p. 476) ; et c'est bien l'apparition de l'homme, ce "porteur de la Marque sombre maudite » dans Dark Souls, qui constitue l'un des centres de gravité de ces récits. Néanmoins le problème est plus épineux dans ce dernier jeu, où l'Âge du Feu, qui marque la naissance de l'homme, est lui-même un Âge cyclique, voué à la répétition - ce que l'on retrouve également dans Bloodborne avec les périodes de chasse. Cette dimension cyclique de la temporalité historique est peut-être ce qui confère à la série cet inachèvement transitionnel dont résulte l'imbrication entre mythe et religion - ce qui pourrait être lié, sur un plan générique, à la perspective d'une fantasy indissociable d'un passé éternisé voué à la répétition cyclique du même (Besson, 2007, p. 158). Les paroles du PNJ Straid d'Olaphis dans Dark Souls 2 sont éclairantes à ce propos, en ce qu'elles illustrent toute la confusion 
entre mythe et histoire, croyance et expérience, mémoire collective, individuelle et oubli :

Bien des royaumes ont vu le jour avant de disparaître sur ce petit coin de terre ; le mien n'était pas le premier. Tout ce qui a un commencement a une fin. Nulle flamme, aussi brillante soit-elle, ne brûle éternellement. Mais un jour... de ses propres cendres, elle renait, et un nouveau royaume voit le jour... La malédiction ne connait pas de fin ! Hé hé hé ! Et votre chair maudite va désormais hériter de la flamme. Hé hé...

On doit la pyromancie à la sorcière ancestrale du Feu. Elle a absorbé la puissance de la flamme, avant de donner naissance à cet art. Cette sorcière serait entrée en guerre contre les Anciens aux côtés de ses filles. Mais ce ne sont là que des légendes. À quel point pouvez-vous croire à ces choses - là ? Hé hé...

\section{Quelques considérations herméneutiques}

Nombre de problèmes de nature herméneutique, marque de la richesse interprétative $\mathrm{du}$ jeu au détriment, comme on l'a vu, de son aspect narratif, pourraient être considérés. Nous en avons soulevé quelques-uns.

L'un des problèmes les plus manifestes concerne le statut de la transcendance des entités divinisées, thématique recouvrant l'entièreté de la série. Celle-ci semble nous fournir une réponse plus ou moins claire: il n'y a pas quelque chose de tel qu'une transcendance véritable, au sens épistémique kantien comme au sens ontologique de ce qui dépasse les limites de l'univers dans lequel évolue le joueur. Ce qui semble transcendant au joueur-interprète, ou ce qui se donne pour transcendant, n'est jamais qu'un processus de manipulation et/ou d'instrumentalisation des hommes visant à assurer la perpétuation d'entités puissantes, mais dans le même temps, vulnérables et périssables - ce qui pourrait engager une critique formelle du pouvoir théocratique.

Dans Bloodborne, les Grands peuvent être assimilés dans un premier temps à des créatures infiniment supérieures similaires, dans la folie qu'elles inspirent, aux Grands Anciens de Lovecraft. Toutefois, il s'avère que celles-ci sont vouées à l'extinction et qu'il leur faut s'unir à des humains pour garantir leur propre perpétuation. Inversement, l'être humain lui-même peut parvenir à la connaissance des Grands, voire à devenir l'un d'entre eux à l'issue du jeu. Ici, c'est le savoir même qui est frappé du sceau du sacré : cette quête de la connaissance interdite s'exprime d'un point de vue ludique lorsque le joueur augmente sa lucidité via l'affrontement avec des chefs notamment, ce qui lui permet, entre autres, de percevoir ces créatures invisibles dispersées çà et là dans certaines zones du jeu. Dans le même temps, un taux trop élevé de lucidité le rend davantage sensible à l'altération d'état qualifié de "folie». On peut en déduire que la connaissance n'est pas tant le pouvoir de nous guider vers la Vérité divine que la découverte de nature ontologique d'un Monde sans Dieu où l'homme est livré à lui-même parmi des êtres cauchemardesques. D'un point de vue métatextuel, il pourrait également renvoyer, de manière plus savoureuse, à l'état du joueur-interprète qui, désirant à bon droit faire toute la lumière sur la mythologie, se perd dans des circonvolutions insurmontables (le statut de folie) ou qui découvre l'essentielle incomplétude du texte.

Dans la saga Dark Souls, le problème de la transcendance n'est pas tant épistémologique que théologique : il concerne avant tout la Première Flamme à l'origine de la disparité du Monde, augurant d'un point de vue mythologique l'Âge du Feu et le terme de l'Âge 
intemporel des Dragons. Cette Première Flamme est, de manière cyclique, vouée à l'extinction si elle n'est pas ravivée par des Âmes. Elle apparaît dans le texte comme une substance dont l'hypertextualité la rapprocherait du Buisson Ardent de la tradition biblique dans sa dimension théophanique, ou encore une manifestation empirique du feu héraclitéen - principe ontologique chez le philosophe grec. Cette Première Flamme peut se consumer sans jamais disparaître intégralement, comme le sous-entend le PNJ Gardienne du Feu dans Dark Souls 3 : « La Première Flamme se meurt. Notre monde sera bientôt la proie des Ténèbres... Mais un jour, nous verrons y danser les lueurs rayonnantes de petites flammes... Des lueurs évoquant la braise... ». De plus, elle requiert des sacrifices pour être attisée. Cette caractéristique est intéressante dans la mesure où, contrairement à la tradition vétérotestamentaire, ce qui relèverait du divin dans la saga Dark Souls et qui pourrait s'apparenter symboliquement au pneuma (c'est-àdire le Saint-Esprit) n'est pas éternel ; il ne peut persister que s'il est réanimé, autrement dit si la foi en lui est préservée.

Enfin, ceux que l'on appelle « dieux » dans Dark Souls n'ont pas vraiment eux-mêmes de caractère transcendant. Ils ne sont jamais qu'une race différente de celle des hommes comme le sont les géants de Dark Souls 2. Néanmoins, si nombre d'entre eux font l'objet de cultes, leur royaume d'Anor Londo, se révèle quasiment déserté. ${ }^{11} \mathrm{Si}$ certains veillent à la sauvegarde de la Première Flamme, la plupart d'entre eux ont quitté la scène, ce qui illustre l'idée de Jean-Louis Siran selon laquelle le mythe a trait «à la croyance de l'autre en tant que j'ai moi-même le sentiment d'échapper à ses illusions » (Siran, 1998, p. 56). Les hommes prient littéralement pour rien ou pour personne; la foi est à présent vide de sens - en témoignent certains ennemis qualifiés de carcasses (sortes de zombies) se livrant dans Dark Souls 3 à des prières conçues ici comme répétition mécanique du même vidée de toute spiritualité.

\section{Conclusion}

51 Établir un joueur modèle qui joue le jeu de l'interprétation permet de révéler les problématiques du religieux et du mythologique. En effet, analyser la progression du joueur profane à l'interprète met en lumière des paradoxes ludonarratifs, des interrogations sur la nature des êtres croisés par l'avatar, mais aussi des mondes pour lesquels la vivacité de la mythologie est le reflet d'une religion sur le déclin. La religion et le mythologique sont ainsi les leviers principaux qui vont articuler la plupart des interprétations du joueur. En acceptant de jouer le jeu de Miyazaki, le joueur modèle s'engage ainsi dans une quête herméneutique sans fin.

Il y aurait comme un malentendu à vouloir mettre sur pied une herméneutique qui rendrait compte de manière exhaustive de l'univers fictionnel. Cette entreprise reste à jamais inachevée et sans doute vaine - mais absolument pas dénuée d'intérêt ludonarratif - en vertu du fait que la dimension fragmentaire annihile la possibilité même de l'unité diégétique: "on doit dire qu'à cette unité, le ou les fragments ne renvoient pas dans la mesure où, dans le fragment, l'unité est justement brisée et défaite » (Frangne, 2005).

53 L'ensemble de ces phénomènes interprétatifs et l'éclatement de cette diégèse expliquent sans doute l'effervescence en ligne autour du jeu (domaine participatif) où nombre de contributeurs - de YouTube aux forums de jeux vidéo - s'affairent à expliquer ces mondes, à rendre compte de leur cohérence. Dans une certaine mesure, 
notre approche pourrait être ainsi poursuivie par une étude sur les publics et la communauté qui se solidarise autour du lore des Soulsborne: comment s'organisentelles? Quels sont les prismes culturels et sociétaux qui influencent de telles interprétations?

\section{BIBLIOGRAPHIE}

ARSENAULT D. \& PERRON B. (2009) «In the Frame of the Magic Cycle. The Circle(s) of Gameplay » in B. Perron \& J. P. M. Wolf, The Video Game Theory Reader 2, New York, Routledge, pp. 109-131.

BESSON A. (2007) La fantasy, Paris, Klincksieck.

CLEMENT F. (2013) «Demon's Souls : L'esthétique de la répétition au cœur de la pratique hardcore du jeu vidéo ", Kinephanos, vol. 4, 1, pp. 49-63.

DE LAUNEY M. (2010) « Genèse 1 - Une cosmogonie ? », Revue d'Histoire et de Philosophie religieuses, 90-4, pp. 465-477.

DI FILLIPO L. (2012) « La dichotomie chercheur-joueur dans la recherche en jeu vidéo : pertinence et Limites ", in L. Di Filippo, A. Michel \& H. François (dirs.), La Position du doctorant. Trajectoires, engagements, réflexivité, Nancy, Presses universitaires de Nancy, pp. 171-192.

DOR S. (2017) « Des conceptions cycliques de la cognition en temps réel », Parenthèse vidéoludique https://www.simondor.com/blog/2017/03/conceptions-cycliques-cognition-en-temps-reel.html DURKHEIM E. (1991 [1912]) Les Formes élémentaires de la vie religieuse, Paris, Le Livre de poche.

ELIADE M. (1965) Le Sacré et le Profane, Paris, Gallimard.

FAVARD F. (2015) La Promesse d'un dénouement ; Enigmes, quêtes et voyages dans le temps dans les séries télévisées de science-fiction contemporaines, thèse de doctorat, Université Bordeaux Montaigne. GENETTE G. (1982) Palimpsestes. La Littérature au second degré, Paris, Éditions du Seuil.

GENVO S. (2008) «Caractériser l'expérience du jeu à son ère numérique : pour une étude du " play design " " http://www.expressivegame.com/publications/colloques/caracteriserlexperience-du-jeu-a-son-ere-numerique-pour-une-etude-du-play-design-s-genvo/.

GENVO S. (2011) « Jeux Vidéo », Communications, 88, pp. 93-101.

GRODAL T. (2000) «Video Games and the Pleasures pf Control », in D. Zillmann \& Vorderer P. (dir.), Media Entertainment: the Psychologie of its Appeal, N.J, Lawrence Erlbaum Associates, pp. 197-213.

HUIZINGA J. (1988 [1938]) Homo Ludens. Essai sur la fonction sociale du jeu, Paris, Gallimard.

JENKINS H. (2004) « Game Design as Narrative Architecture », in N. Wardrip-Fruin \& P. Pat Harrigan (dir.), First Person, New Media as Story, Performance, and Game, Cambridge, The MIT Press.

KING G., KRZYWINSKA T. \& SIEGEL G. (dir.) (2002) Screenplay: Cinema/videogames/interfaces, New York, Wallflower Press. 
LABRUDE G. (2018) Dark Souls et l'obscur cheminement vers le mythe: Nostalgie de l'Enfer, syncrétisme, hiérophanie et mise en scène, communication au colloque « ce que la remédia(tisa)tion fait au mythe : de la modernité à aujourd'hui », Université de Québec à Montréal https:// www.academia.edu/36427712/

Dark_Souls_et_lobscur_cheminement_vers_le_mythe_Nostalgie_de_l_Enfer_syncr \%C3 \%A9tisme_hi \%C3 \%A9rophanie_et_mise_en_sc

LEAVITT J. (2005) « Présentation : le mythe aujourd'hui. » Anthropologie et Sociétés, 29-2, pp. 7-20.

MACWHERTOR M. (2019) « Why Demon's Souls is the most important game of the decade ", Polygon, https://www.polygon.com/2019/11/15/20967134/demons-souls-most-important-gameof-the-decade.

MALET A. (1962) Mythos et Logos, la pensée de Rudolph Bultmann, Genève, Labor et Fides.

MECHERI D. \& ROMIEU S. (2017) Dark Souls. Par-delà la mort : Volume 1 - Demon's Souls, Darks Souls et Dark Souls II (RPG), Toulouse, Third Editions.

PERRON B. (2013) «L'attitude ludique de Jacques Henriot », Sciences du jeu, 1, http:// journals.openedition.org/sdj/216

RIALLAND I. (2005) « Mythe et hypertextualité », Fabula, https://www.fabula.org/atelier.php? Mythe_et_hypertextualit \%26eacute \%3B.

RYAN M-L. (2004) Narrative accross Media: The langages of Storytelling, Lincoln, University of Nebraska Press.

SALEN K. \& ZIMMERMAN E. (2004) Rules of Play: Game Design Fundamentals, Cambridge, The MIT Press.

SAUTE-REQUIN (2019) « La sérialité aux bords de la fiction télévisée, ou l'intermédialité comme mise en crise du " tout narratif" sériel », TV/Series, 15, http://journals.openedition.org/tvseries/ 3724

SIRAN J-L. (1998) L'illusion mythique, Paris, PUF.

\section{NOTES}

1. Certains journalistes et joueurs, en lien avec les différentes similarités liées au gameplay et à la narration entre les jeux, ont été amenés à attribuer à la série l'appellation de Soulsborne, dont il nous a malheureusement été impossible de retracer l'origine exacte.

2. Puyo, "Test : Demon's Souls, le venin de la nouvelle génération ", Gamekult.com, 13/11/2020 https://www.gamekult.com/jeux/demon-s-souls-3050882349/test.html, consulté le 31 mai 2021.

3. Michaeal McWhertor, "Why Demon's Souls is the most important game of the decade", Polygon 15/11/2019, https://www.polygon.com/2019/11/15/20967134/demons-souls-mostimportant-game-of-the-decade,

consulté le 08 février 2021. Les traductions françaises des textes anglais cités dans l'article sont le fait de ses auteurs.

4. Ayden, « Dark Souls : Références culturelles d'hier et d'aujourd'hui », jeuxvideo.com, 31/03/2016, https://www.jeuxvideo.com/dossier/464310/dark-souls-references-culturelles-d-hier-et-daujourd-hui/464170.htm, consulté le 17 mai 2021.

5. Keza Macdonald « The Mind Behind Dark Souls », IGN.com, 13/01/2018 https://www.ign.com/ articles/2011/11/08/the-mind-behind-dark-souls, consulté le 26 février 2021.

6. Simon Parkin, «Interview- Bloodborne creator Hidetaka Miyazaki: "I didn't have a dream. I wasn't ambitious." ", Theguardian.com, 31/03/2015 https://www.theguardian.com/technology/ 
2015/mar/31/bloodborne-dark-souls-creator-hidetaka-miyazaki-interview,

dernière consultation le 5 mai 2020.

7. Avec Bloodborne et Dark Souls 3, le brouillard apparaît a posteriori. Il faut en effet atteindre la zone du chef au préalable et observer dans un deuxième temps l'entrée de l'arène pour déclencher son apparition ; le brouillard persiste d'ailleurs à la suite d'une défaite contre ledit boss.

8. Maiden dans la traduction anglaise. Le qualificatif est étrangement occulté dans la version française du jeu, dans laquelle Maiden Astrea est retraduite Astrée. Ce détail est d'autant plus important qu'en raison d'un souci de cohérence en lien avec son inspiration occidentale, ce jeu japonais ne dispose originellement que d'un doublage anglais (Mecheri et Romieu, 2017, p. 40-41). 9. La description de l'anneau de l'aîné solaire dans Dark Souls 3, dans lequel l'objet figure aussi, diffère légèrement : le pronom indéfini neutre " on » est remplacé par le terme " histoire ", ce qui accentue la dimension mythologique - au sens, ici, de ce qui n'est pas inclus dans la trame historique et la Tradition œcuménique - d'un tel accessoire.

10. Rappelons ici la définition de l'hypertextualité que propose Gérard Genette dans Palimpsestes : "J'entends par là toute relation unissant un texte $B$ (que j'appellerai hypertexte) à un texte antérieur A (que j'appellerai, bien sûr, hypotexte) sur lequel il se greffe d'une manière qui n'est pas celle du commentaire " (Genette, 1992, p. 13).

11. Afin de dissiper l'illusion d'Anor Lando, il est nécessaire d'agresser la déesse Gwenevere, PNJ que l'on rencontre au cœur de cette cité. Suite à l'attaque, Gwenevere s'évanouit sans augmenter notre compteur d'âmes, et le royaume jusqu'à là étincelant sous le soleil est plongé dans l'obscurité. Dans la diégèse, ce que nous avons identifié comme la déesse de la fertilité n'était qu'une fantasmagorie née de la magie de son frère, Gwendolin bien réel quant à lui et dissimulé derrière un second type d'illusion (un passage caché).

\section{RÉSUMÉS}

Affronter le problème de la présence des mythes et du religieux au sein de la série Soulsborne désignation commune aux derniers jeux du développeur From Software - dans laquelle les motifs et renvois hypertextuels s'y référant abondent, présuppose de s'être livré à un examen psychocognitif du joueur modèle souhaité pour ces jeux. Une telle analyse nous permet de mettre en perspective l'importance fondamentale de la capacité herméneutique d'un tel joueur, confronté à l'absence de référent narratif. Nous soutenons en cela que les dimensions religieuses et mythologiques demeurent les leviers fictionnels centraux à disposition du joueur, et dont il convient de dessiner les contours afin d'appréhender et d'interpréter ces univers diégétiques.

Facing the challenge of myths and religious within the Soulsborne serie - shared designation including the last video games of From Software - in which hypertextual motifs and references abound, imply to have conducted a psycho-cognitive examination of a wished ideal player in these games. Such a study allows us to highlight the fundamental importance of the hermeneutic ability of such a player confronted to the lack of narrative referent. We argue that the religious and mythical dimensions are central fictionnal levers and at the diposal of the player. It is then required to draw the outlines of such levers in order to understand and to interpret these diegetic worlds. 
INDEX

Keywords : religion, mythology, hermeneutic, interpretation, series, Environmental Storytelling, heuristic/hermeneutic spirals, ideal player

Mots-clés : religion, mythologie, herméneutique, interprétation, série, narration environnementale, spirales heuristiques/herméneutiques, joueur modèle

\section{AUTEURS}

YANNICK KERNEC'H

Université Rennes 2

CLÉMENT PERSONNIC

Université de Montréal 